\title{
The Tumor Immune Contexture of Prostate Cancer
}

\author{
Natasha Vitkin ${ }^{1}$, Sarah Nersesian ${ }^{1,2}$, David Robert Siemens ${ }^{1,3}$ and Madhuri Koti 1,2,3,4* \\ ${ }^{1}$ Department of Biomedical and Molecular Sciences, Queen's University, Kingston, ON, Canada, ${ }^{2}$ Cancer Biology and \\ Genetics, Queen's Cancer Research Institute, Kingston, ON, Canada, ${ }^{3}$ Department of Urology, Queen's University, Kingston, \\ ON, Canada, ${ }^{4}$ Department of Obstetrics and Gynecology, Queen's University, Kingston, ON, Canada
}

OPEN ACCESS

Edited by:

Leila Akkari,

The Netherlands Cancer Institute

(NKI), Netherlands

Reviewed by:

Ekaterina Jordanova,

Center for Gynaecologic Oncology

Amsterdam, Netherlands

Zong Sheng Guo,

University of Pittsburgh, United States

${ }^{*}$ Correspondence:

Madhuri Koti

kotim@queensu.ca

Specialty section:

This article was submitted to Cancer Immunity and Immunotherapy,

a section of the journal

Frontiers in Immunology

Received: 27 December 2018 Accepted: 07 March 2019

Published: 28 March 2019

Citation:

Vitkin N, Nersesian S, Siemens DR and Koti $M$ (2019) The Tumor Immune

Contexture of Prostate Cancer.

Front. Immunol. 10:603.

doi: 10.3389/fimmu.2019.00603
One in seven men in North America is expected to be diagnosed with prostate cancer (PCa) during their lifetime $(1,2)$. While a wide range of treatment options including surgery, radiation, androgen deprivation and chemotherapy have been in practice for the last few decades, there are limited treatment options for metastatic and treatment resistant disease. Immunotherapy targeting T-cell associated immune checkpoints such as CTLA-4, PD-L1, and PD-1 have not yet proven to be efficacious in PCa. Tumor mutational burden, mutations in DNA damage repair genes, immune cell composition and density in combination with their spatial organization, and expression of immune checkpoint proteins are some of the factors influencing the success of immune checkpoint inhibitor therapies. The paucity of these features in PCa potentially makes them unresponsive to contemporary immune checkpoint inhibition. In this review, we highlight the hallmark events in the PCa tumor immune microenvironment and provide insights into the current state of knowledge in this field with a focus on the role of tumor cell intrinsic events that potentially regulate immune related events and determine therapeutic outcomes. We surmise that the cumulative impact of factors such as the pre-treatment immune status, PTEN expression, DNA damage repair gene mutations, and the effects of conventionally used treatments on the anti-tumor immune response should be considered in immunotherapy trial design in PCa.

Keywords: prostate cancer, tumor immune microenvironment (TIME), immunotherapy, immune checkpoint, DNA damage response, PTEN, hormone therapy

\section{INTRODUCTION}

Prostate cancer $(\mathrm{PCa})$ is the second most commonly diagnosed malignancy in men; each year, $\sim 220,000$ men in the United States are diagnosed with PCa (3). Newly diagnosed PCa is assessed using a combination of typical cancer staging (TNM), histological characteristics of a prostate biopsy, as well as prostate specific antigen (PSA) levels (4). In men diagnosed with lower risk, localized cancer, treatment options include active surveillance, radical prostatectomy (RP) or radiation therapy (RT) (4). Those with higher risk but still potentially curable disease will often require multiple interventions including $\mathrm{RP}+/-\mathrm{RT}$, as well as androgen deprivation therapy (ADT) as an adjuvant (4). However, these treatments are not curative for all patients, and biochemical recurrence occurs in approximately $25 \%$ of patients (5). Following recurrence, or for those presenting with metastatic disease, $\mathrm{ADT}$ is the current standard of care to remove circulating androgens that drive PCa growth and survival (6). Despite an initial clinical response, the majority of patients fail ADT and develop castration-resistant PCa (CRPC), a state of disease 
progression which occurs despite surgical or medical castration (7). Short term responses to systemic chemotherapy or other androgen receptor targeted therapies may occur, however, CRPC is ultimately lethal and results in the death of $\sim 29,000$ American men each year $(3,7)$. The high morbidity of this disease urgently necessitates the development of novel treatment strategies.

One such promising approach under investigation for PCa therapies is immunotherapeutic treatments that harness and exploit the body's intrinsic anti-tumor immune response. The recent success of immune checkpoint inhibitors (ICIs) in cancers such as melanoma and bladder cancer, has led to renewed interest in the tumor immune contexture to identify prognostic and predictive biomarkers as well as to direct novel immunotherapy combinations and sequencing toward precision cancer therapies (8). Several investigations on spatial and molecular profiling of tumors have attempted to define a pan-cancer immune landscape ranging from broad classifications as immunologically cold or hot (9), to six molecular subtypes; wound healing, interferon (IFN)- $\gamma$ dominant, inflammatory, lymphocyte depleted, immunologically quiet, and transforming growth factor (TGF) $\beta$ dominant (10). Such comprehensive classification of the tumor immune microenvironment (TIME) in prostate cancer $(\mathrm{PCa})$ is currently unavailable. Attributed to the disease complexity and significant heterogeneity, a deeper view of the PCa TIME is currently lacking and is needed to inform the design of immunomodulatory treatments and drug sequencing. In-depth knowledge regarding the cumulative effects of oncogenic drivers in distinct TIME states is critical to guide selection of therapies exploiting the anti-tumor immune responses. In this review, we focus on the immune features associated with localized and metastatic PCa to allow a knowledge-driven approach for future immunotherapybased treatments.

\section{The PCa Tumor Immune Microenvironment (TIME)}

Immune responses, involving both secreted and cellular factors in the TIME, can drastically impact the balance between tumor progression, tumor clearance, and treatment response. Specifically, the variability in response has shifted the focus of rational design of ICIs to incorporating the features of the TIME such as infiltration and localization of tumor infiltrating lymphocytes (TILs) and presence of immunosuppressive cell populations (11). Interestingly, among the genitourinary cancers, PCa exhibits a unique TIME profile with distinct features of these populations (12).

The presence of cytotoxic and helper $\mathrm{T}$ lymphocytes within tumor margins has been associated with favorable prognoses and clinical implications across a multitude of cancer types (13). Identifying the critical function of TILs in cancer progression led to the establishment of the "immunoscore" as a standardized metric to assess the tumor immune contexture based on the density and location of CD3+ and CD8+ T cells (13). Given that the compartmentalization of TILs within the tumor is a critical feature associated with response and outcomes, only TILs within the tumor center and invasive margins are considered in the immunoscore (14). Using this classification in combination with tumor inflammation signature, solid tumors can be broadly classified into T cell inflamed/ "hot," and non-T cell inflamed/ "cold" tumors (15). ICI trial outcomes in some solid cancers such as melanoma urothelial and lung cancer, show that favorable responses are observed in hot tumors, which have a pre-existing higher density of TILs and expression of an IFN-associated gene signature $(8,16)$. Patients with an inflamed TIME also exhibit better responses to traditional therapies such as radiation and chemotherapy. Both treatment strategies are known to stimulate immunogenic cell death and consequently enhance the efficacy of immune checkpoint inhibitor therapy $(14,17)$.

In many solid tumors, high CD8+ TIL infiltration, especially their activated state, correlates with better prognosis due to their cytotoxic functions $(18,19)$. However, the prognostic relevance of CD8+ TILs is unclear in PCa, with some studies demonstrating that a high tumor TIL infiltrate is detrimental to patient survival. Indeed, one study reported that a higher density of stromal CD8+ TILs associates with poor prognosis in radical prostatectomy specimens and demonstrated a significant correlation between immunosuppressive CD73 expression and CD8+ TIL density (20). Another report showed that infiltration by CD8+ TILs within the invasive margins and stromal compartment of tumors associates with poor clinical outcomes and a shorter time until BCR in PCa patients (21). Another study evaluated tumor infiltrating CD8+ TILs and programmed death-ligand 1 (PD-L1) immune checkpoint expression in 51 node-positive PCa samples and reported that both CD8+ TIL density and PD-L1 expression were independent predictors of clinical progression (22). Most recently, an analysis of gene expression profiles of 1,567 prostatectomy specimens showed that high tumor TIL infiltrates were associated with worse distant metastasis-free survival (23). These findings may be due to improper TIL functionality; previous studies suggest that CD8+ TILs in the PCa TIME may be dysfunctional or suppressed, contributing to impaired cytotoxic responses despite tumor antigen stimulation $(21,24)$. It is currently unknown whether PCa-infiltrating TILs are in a state of anergy, exhaustion, or senescence; all of these are characterized by low or negligent levels of effector function (25). Further research is needed to characterize the functional status of TIL infiltrates in PCa to definitively assess the impact of their localization on prognosis.

The immune response is a balance between immunostimulatory and immunosuppressive factors; accordingly, functional TIL activity in PCa could be impaired by the magnitude of impact of secreted and cellular immunosuppressive factors. When looking at other T-cell populations in PCa, studies have noted high proportions of both CD4+ and CD8+ forkhead box P3 (Foxp3+) regulatory T cells (Tregs), within the tumor margin and epithelial compartment in $\mathrm{PCa}(26,27)$. Another report examining changes in TIL infiltrates in PCa biopsies at diagnosis and subsequent relapse showed that increased infiltrates of Foxp3+ TILs were significantly associated with worse progression-free survival and overall survival (28). Preliminary data suggests that the presence of other receptors such as CCR4 on Tregs may impact PCa patient survival, although further research is required to support this 
claim (29). Previous reports in gastric cancer show the positive association of CD8+Foxp3+ $\mathrm{T}$ cells with favorable prognosis which is in contrast to findings in PCa (30). In addition to the presence of immunosuppressive lymphocytes, multiple reports have demonstrated that high tumor-associated macrophage (TAM) infiltration in the PCa TIME is pro-tumorigenic (31), however, most do not differentiate between the M1 (tumor suppressive) and M2 (tumor promoting) phenotypes of TAMs. Notably, co-culturing of naïve monocytes with PCa cells resulted in decreased expression of co-stimulatory molecules and reduced endocytic ability compared to monocytes stimulated with M-CSF (31). Furthermore, these macrophages secreted high levels of M2-associated immunosuppressive cytokines and chemokines, with TGF- $\beta 2$ being the most highly expressed (31). Given the established role of TGF- $\beta$ in immune exclusion, this may be one of many factors contributing to poor TIL infiltration in PCa (32). In addition to providing insights into the association between M2 macrophages and poor prognosis in $\mathrm{PCa}$, the immunosuppressive role of TGF- $\beta$ is critical in the context of current ICI, where targeting TGF- $\beta$ prior to ICI treatment has been suggested as an approach to improve response (32).

\section{Factors Affecting the PCa TIME}

The factors underlying evolution of an immunologically cold PCa TIME may be attributed to hormonal influence, genetic alterations, selective pressures of treatment. Further, immune exclusion and/or evasion mechanisms as a result of malignant progression could also lead to a cold TIME state (33). Several tumor intrinsic factors contribute to the evolution of a unique pre-treatment TIME in PCa, in addition to host physiological factors such as age and hormones. Low tumor-associated antigen expression, DDR defects, decreased MHC Class I expression, loss of PTEN protein, and dysfunctional IFN1 signaling are some of the mechanisms thought to be important in determining the features of the PCa TIME (Figure 1).

\section{Tumor Mutational Burden}

A feature of PCa important to the immune landscape is its relatively low somatic mutation burden and consequently diminished neoantigen expression compared to many other cancers (34). Overall rates of mutation in PCa cells are low; one study revealed a mean mutation frequency of 0.9 per megabase, about 10 times lower than that of melanoma (35). A lack of tumor neoepitopes is associated with reduced immune cell attraction to the tumor site, with fewer tumor-specific epitopeMHC interactions, resulting in reduced antigen presenting cells (APCs) cross-priming to TILs. The lack of these key interactions underlies the evolution of a non-inflamed TIME. In this scenario, transformed cells could evade immune cellmediated elimination and proliferate freely (36). Consequently, treatment with immunotherapies would be ineffective as a preexisting active immune contexture would be lacking. Indeed, ICI therapies such as those targeting the PD-L1/PD-1 immune checkpoint axis have the largest clinical impact in cancers with the highest numbers of somatic mutations such as melanoma and non-small cell lung cancer (37).

\section{DDR Gene Defects}

DDR is an important cellular pathway initiated to drive timely and accurate repair of genetic material damaged by mutagens such as ionizing radiation. Lack of cellular DDR mechanisms can lead to the accumulation of genetic aberrations, resulting in tumor evolution and progression (38). While fostering genetic instability, these alterations are also thought to skew the TIME toward an inflamed state, partly by increasing interactions of tumor-specific antigens with infiltrating immune cells or through altering cellular IFN pathways (39). The field of DDR in PCa is relatively understudied because of its low prevalence in this cancer. However, recent next generation sequencing based profiling efforts from The Cancer Genomic Atlas Network highlight these defects in both primary and advanced PCa (40). This study, conducted on primary PCa and localized disease, showed the presence of mutations in the DDR genes BRCA2, BRCA1, CDK12, ATM, FANCD2, RAD51C in 19\% of cases (40). Similarly, an enrichment in DDR gene mutations in the metastatic scenario was reported in $23 \%$ of cases (41). Analyses based on 150 primary and mCRPC cases showed an enrichment in aberrations in TP53 (53\%), RB1 (21\%), the PTEN$P I 3 K$ pathway $(49 \%)$, and $A R(63 \%)$ in mCRPC compared to localized disease (41). The presence of many molecular subtypes with different mutations in DDR pathways and driver mutations makes generalizing the TIME status in patients challenging (Figure 2).

In line with these observations, a recent trial reported that PCa patients with DDR deficiencies (BRCA1, BRCA2, and ATM) had significantly better responses to Olaparib with corresponding increases in overall survival and progression free survival (42). No differences in these metrics were reported between patients with germline mutations compared to somatic aberrations, suggesting that by the time CRPC occurs, the impacts of germline and somatic DDR defects are functionally equivalent. In localized $\mathrm{PCa}$, percentage of men with germline DDR defect was lower (4.6\%), and odds ratios also support a higher proportion of DDR defects in men with mCRPC compared to localized PCa (43). These results are especially promising for patients who have failed multiple treatments, as they implicate late stage PCa patients with DDR deficiency as better responders to therapy. In a study of over 600 mCRPC cases, $11.8 \%$ had a germline mutation in a prominent DDR gene, compared to only $4.6 \%$ in localized PCa patients (43). Furthermore, the presence of germline mutations in BRCA2, ATM and CHEK2 were associated with histologically advanced disease (43). The challenges of mapping the primary and metastatic sites make it difficult to assign a clear trajectory of these events as secondary to treatment pressures vs. progression of an inherently aggressive cancer.

It has been established that DNA damage induces AR activity, which feeds back to activate gene expression program promoting DNA repair; both in vitro and in vivo, activating AR signaling can promote resistance to DNA-damaging agents (44). Synergistic effects of second-generation ADT and radiotherapy to decrease PCa cell survival has been shown to be mediated partly by PARP1 (45). Since recurrent PCa is treated with ADT, sensitizing tumors to radiotherapy is common, however, it may also contribute to 


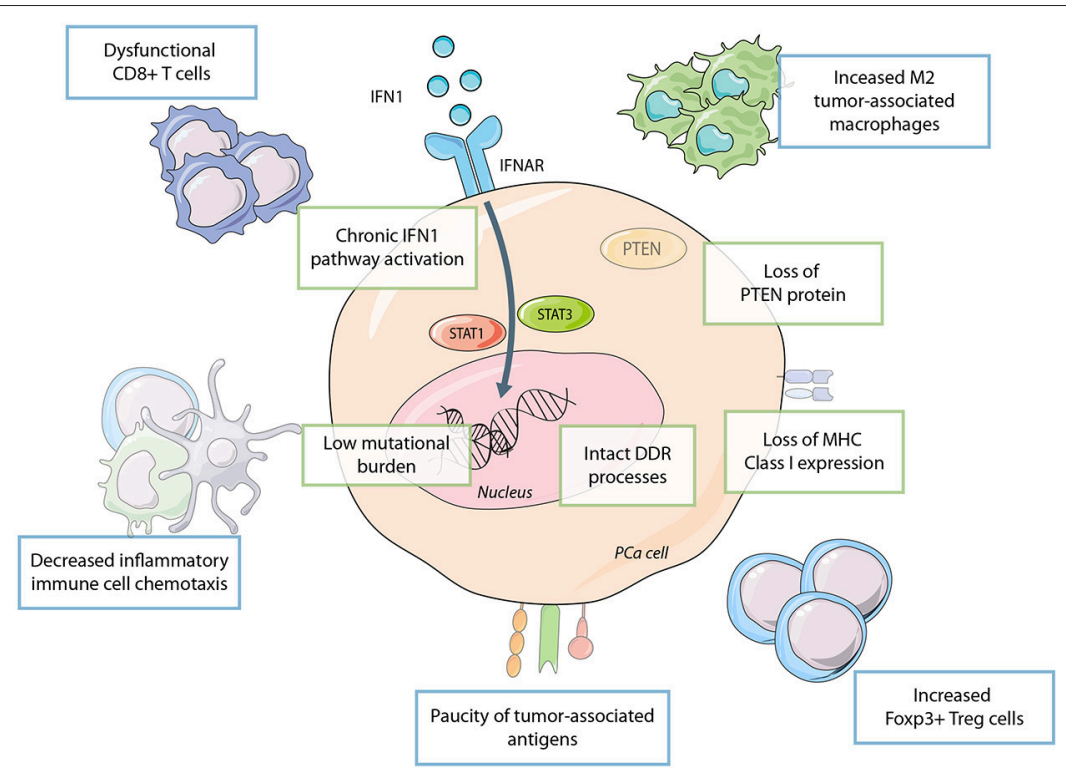

FIGURE 1 | Schematic illustrating the various factors impacting the PCa TIME and their propose on the TIME. Numerous cancer cell-intrinsic factors drive the evolution of a the heterogenous pre-treatment TIME. In PCa, loss of PTEN could lead to a dysfunctional IFN1 signaling. Other features include low tumor-associated antigen expression, decreased MHC Class I expression, which could potentially contribute to immune escape. These, in combination with the pre-existing immune contexture led by other factors, are thought to be critical in determining the balance between activated and suppressive states of TILs including classical or alternatively activated tumor-associated macrophages.

clonal evolution and newer mutations. Regardless, as seen in other cancers $(32,46)$, DDR defects are indeed beneficial from an immune perspective and could potentially form the basis for immune sensitization of PCa to ICIs.

\section{Loss of MHC / HLA Expression}

MHC Class I proteins are normally expressed on nucleated cells and present cytosolic peptides to $\mathrm{T}$ lymphocytes, triggering an immunostimulatory signal cascade resulting in $\mathrm{T}$ cell proliferation and target cell lysis (47). Accordingly, loss of MHC Class I expression is a common immune evasion mechanism employed by a variety of cancer types (47). Defective MHC Class I may result from aberrations in multiple pathways including HLA synthesis and transport, antigen processing, or loss of critical accessory proteins (47). Preliminary evidence also suggests that epigenetic silencing of MHC Class I genes is important in PCa (48). This loss of MHC Class I expression has been documented in both metastatic PCa cell lines and clinical specimens $(49,50)$. Different signaling pathways including the IFN axis can also impact MHC Class I expression; in a syngeneic mouse model of PCa, treatment with IFN- $\gamma$ led to increased survival and heightened expression of proteins important in MHC Class I production such as TAP1 (51). Cell line experiments have also demonstrated that radiation increases MHC Class I expression and leads to unique MHC Class I binding antigenic peptides (52). Increased MHC Class I expression in tumors is predicted to facilitate the activation and expansion of CD8+ TILs within the invasive margins of the tumor, eliciting a more robust immune response. However, in the context of an immunosuppressive TIME lacking a dense TIL infiltrate, heightened expression of MHC Class I proteins in isolation is unlikely to shift the TIME toward an immunoactive state, especially in cases with concurrent immunosuppressive features.

\section{PTEN Loss}

A well-characterized molecular aberration in PCa is the loss of the tumor suppressor protein PTEN. PTEN is generally known as a lipid and protein phosphatase encoded by the PTEN gene which antagonizes the pro-growth PI3K signaling pathway and is deleted in up to $30 \%$ and mutated in 2$5 \%$ of primary PCa cases (53). Emerging literature suggests that the immune regulatory functions of PTEN are mediated through modulating the activation of cellular IFN1 pathways (54). In other cancers such as melanoma, patients with PTEN loss exhibited significantly poorer responses to PD-1 ICI and had lower TIL infiltration compared to patients with $>10 \%$ of tumor cells positive for PTEN staining (55). Furthermore, the therapeutic activity of tumor-specific TILs from adoptive T cell therapy was significantly reduced in mice with PTENsilenced melanoma cells compared to those with an intact PTEN gene, indicating that PTEN can confer sensitivity to T-cell-based immunotherapy (55). Other alterations may also cooperate with PTEN loss to drive distinct tumor immunological phenotypes. Using in vivo models, a recent study demonstrated the qualitative and quantitative impact of Pten loss in the TIME. Specifically, myeloid-derived suppressor cell (MDSC) infiltrates in Pten ${ }^{-/-} ; \mathrm{Zbtb}_{7} a^{-/-}$prostate tumors exhibited a distinct phenotype affecting NF- $\kappa$ B signaling whereas MDSCs within Pten $^{-/-}$; Tp53 $53^{-/-}$tumors were associated with Treg 


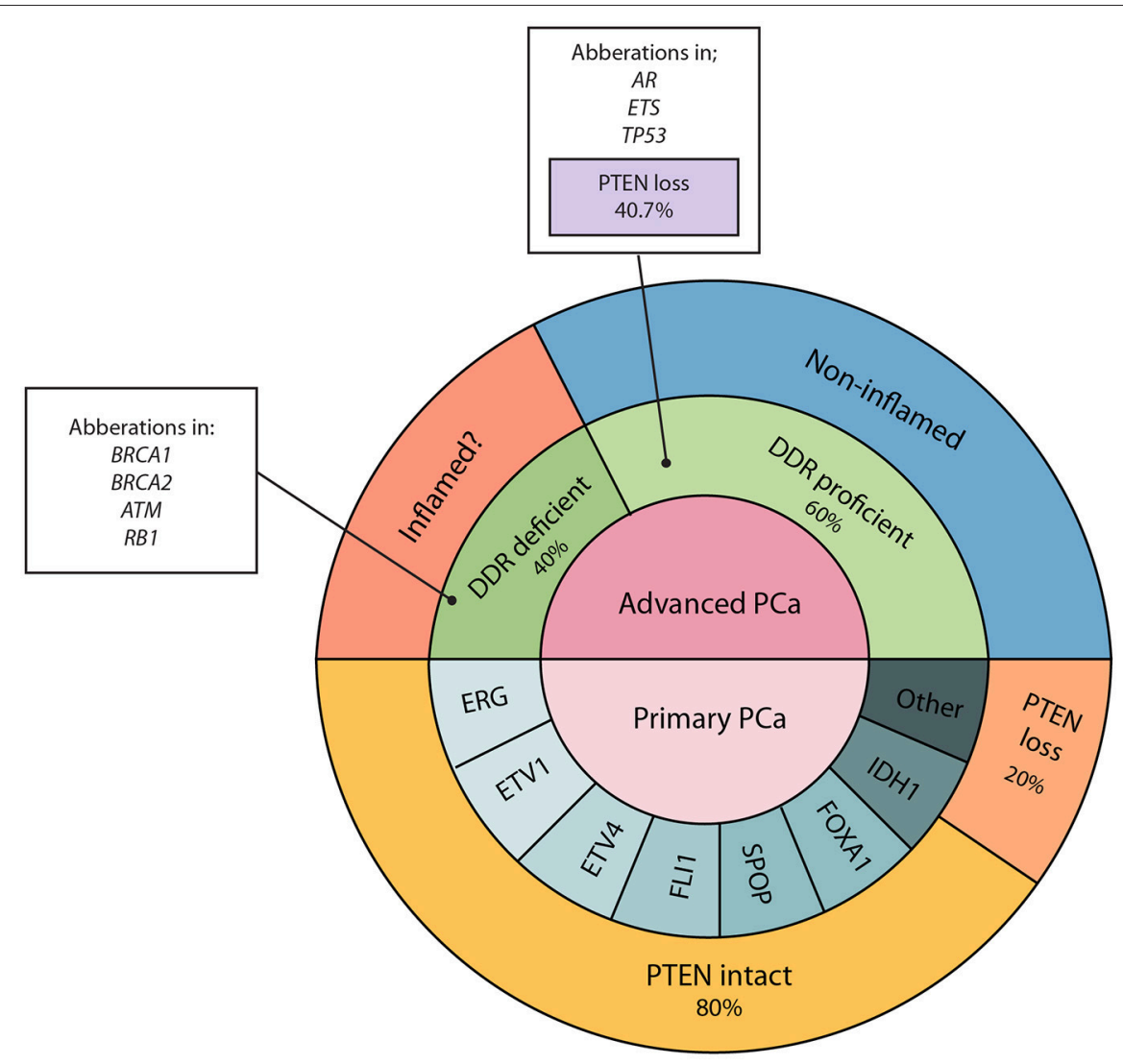

FIGURE 2 | Genetic aberrations associated with primary and advanced PCa. PTEN loss is associated with $20 \%$ primary and $40.7 \%$ of advanced PCa. Increased proportions of mutations in DNA damage repair genes, BRCA1, BRCA2, ATM, and RB1, have mostly been reported in advanced PCa. While DDR deficient tumors may exhibit increased numbers of oncogenic mutations, we speculate that this may result in a more immunogenic phenotype and give rise to an inflamed TIME as observed in some other solid tumors.

immunosuppression (56). These findings implicate PTEN as a tumor suppressor which, in addition to regulating the PI3K-Akt-mTOR signaling network, can govern the tumor immune milieu and response to immunotherapy, however, these findings must be validated in PCa. These data provide compelling evidence for an undefined mechanistic role of PTEN in altering the immune contexture of the PCa TIME. Recent studies conducted in phosphatase inactive PTEN cells have highlighted its phosphatase independent tumor suppressive functions, specifically in DNA repair and apoptosis $(57,58)$. An area relatively understudied in $\mathrm{PCa}$, however, is the specific effect of altered levels of nuclear, cytoplasmic and secreted PTEN proteins in mediating an aggressive disease and immunosuppressed TIME state. Given that all three isoforms of PTEN exert different regulatory functions (59), in processes that alter cancer progression and immune response in the TIME, future investigations should incorporate these in scenarios where PTEN deficiency is not attributed to loss of $10 \mathrm{q}$ region harboring the PTEN gene. A precise definition of these genotype and associated immunophenotype relationships will allow the development of alternate targeted therapies and improved patient stratification.

\section{IFN1 Signaling}

Few studies have characterized the functional status of immune cell populations in the PCa TIME, but preclinical and clinical data supports that IFN1 signaling in the TIME exerts protective anti-tumor effects in PTEN-deficient tumors. IFN1 is an important group of immunostimulatory cytokines released in response to direct binding of IFN1 to its extracellular receptor, or from cellular detection of invading pathogens by innate pattern recognition receptors (60). It is established that IFN1 is crucial to mounting an efficient anti-tumor immune response, which is accomplished by a variety of mechanisms such as cytokine and chemokine production, increasing the expression of immune costimulatory molecules, activating adaptive immune cells, and facilitating CTL killing (61). The activation of transcription factors STAT1 and STAT3 drive canonical IFN1 signaling by mediating the transcription of over 2000 interferon-stimulated genes, which serve a diverse array of functions involved in stimulating and regulating the innate and adaptive immune responses (62). Combined prostate-specific STAT3 and PTEN deficient mice exhibited accelerated cancer progression and metastasis compared to PTEN-deficient mice; these animals had tumors up to six times larger than $P T E N^{-/-}$ 
mice (63). The authors show that these effects are mediated through the ARF-MDM2-p53 axis, and suggest that PTENdeficient tumors cannot effectively activate this axis, resulting in tumor metastasis (63). However, conflicting evidence has demonstrated that STAT3 inhibition results in decreased PCa cell growth and tumor metastasis, both in vitro and animal models of PCa $(64,65)$. Chronic IFN1 signaling has been associated with immunosuppression and therapy resistance; both unphosphorylated STAT1 and STAT3 (U-STAT1/3) can serve as active transcription factors and mediate the expression of specific subsets of ISGs $(66,67)$. The subset of ISGs activated by U-STAT1 after prolonged IFN1 exposure render cancer cells insensitive to radiation and chemotherapy (68) Multiple studies have demonstrated that in addition to contributing to therapeutic resistance, these genes also promote cancer growth and metastasis (69). The ability of IFN1s to modulate the expression of distinct sets of ISGs through differences in signal duration and STAT activation provides a mechanism to account for the opposing roles of IFN1 in immune stimulation and regulation. Furthermore, it is likely that cellular and environmental cues such as PTEN loss, DDR defects, TIL infiltration and activity, and the presence of immunosuppressive factors reflect these divergent findings.

\section{Impact of Therapy on the PCa TIME}

Androgens and their receptors play a critical role in both progression and treatment of PCa. Antagonists of androgen receptor (AR) such as bicalutamide and enzalutamide are therefore widely used as part of ADT therapy in PCa (70). As the immune response is a dynamic process affected by environmental factors, PCa treatments can also affect the tumor immune contexture. Complex mechanisms of androgen blockade mediated effects on the PCa TIME, ranging from thymic enlargement, increased lymphocyte migration, to GABAA receptor mediated off-target effects leading to impaired $\mathrm{T}$ cell priming have been reported (71). Due to the dependency of PCa cells on androgen signaling, ADT treatment results in cancer cell apoptosis, failing to release immunostimulatory signals (72). In a syngeneic murine model, increased CD3+ T cell infiltration in tumors post orchiectomy (surgical castration) with corresponding tumor regression was observed, albeit eventual relapse (71). This response was associated with a thymic T cell wave, which is typically short-lived, and may be accompanied by increases in regulatory immune cell populations (73). Suppression of both cell mediated and humoral immune responses by $\mathrm{AR}$ antagonists (medical castration) has been reported in syngeneic murine models of PCa $(74,75)$. A key finding is the contrasting impact of medical vs. surgical castration on $\mathrm{T}$ cell priming, which is a critical factor in antitumor immune response. While treatment with gonadotrophinreleasing hormone analogs has similar effects as orchiectomy, opposite effects were observed using AR antagonists. Clearly, more longitudinal studies in patients are warranted to define these precise correlations for effective sequencing of AR antagonists and immune based therapies. Similarly, given their predictive importance (76), and expression of PD-L1, defining the TAM phenotypes that associated with pre- and post ADT treated tumors will be crucial for determining the proper sequencing of ICI treatment. Another important question that remains unanswered pertains to how these changes correlate with the pre-treatment TIME states, specifically with regard to stromal and epithelial localization of cytotoxic TILs.

Treatment-induced ICD leads to the release of cancer cell antigens to which the immune system can respond (77). This mediates the influx and activation of dendritic cells (DCs) and TILs, which can facilitate a more robust anti-tumor immune response. Notably, the presence of an active immune contexture predicts a favorable response to chemotherapy, implicating that cells of the TIME are critical for an individual's response to treatment $(72,78)$. Docetaxel, an effective systemic chemotherapy used for men with metastatic CRPC, does not initiate classic ICD although studies suggest that it can augment TIL-mediated tumor killing and decrease MDSC populations $(79,80)$. In a Phase II clinical trial, metastatic CRPC patients receiving a PSA vaccine and subsequent docetaxel had a median progression-free survival of 6.1 months while patients taking docetaxel alone survived 3.7 months (81). These results suggest that while not directly inducing ICD, docetaxel treatment for CRPC patients may potentiate the immune response and mediate an inflamed TIME.

Radiation therapy is another therapeutic modality, utilized for both curative and palliative indications, that also has been demonstrated to have immunomodulatory properties. Radiotherapy has been shown to increase the number and diversity of tumor-specific surface peptides and expression of MHC Class I molecules in a dose-dependent manner, which increased the efficacy of TIL-mediated cancer cell killing (52). Immuno-potentiation may also be attributed to the release of immunostimulatory cytokines and danger-associated molecular patterns (DAMPs) due to radiation exposure (73). The abscopal effects of radiation on distant metastases in PCa have also been documented; metastatic patients who received first-line radiotherapy had significantly higher overall survival compared to patients who did not receive this treatment in one retrospective study (82). It could be hypothesized that these outcomes could be secondary to radiotherapy-instigated immune activation, which would mediate a systemic anti-tumor immune response targeting distant metastases as well as the primary tumor.

Another relatively understudied area in PCa is the difference in TIME profiles in primary tumors compared to metastatic disease. A recent landmark study comparing 150 matched primary and metastatic CRPC reported novel clinically actionable aberrations, including higher frequencies of aberrations in DDR genes such as BRCA1, BRCA2, and ATM (41). Given the availability of tumor molecular profiles from immunologically distinct sites of metastasis in studies such as this, a comprehensive characterization of the spatial and molecular immune profiles of metastatic lesions could provide an improved understanding of immune evasion mechanisms in PCa.

\section{Current State of Immunotherapy in PCa}

Two vaccine-based immunotherapy approaches have shown moderate success in PCa treatment. Sipuleucel-T is a 
personalized treatment constituting the ex vivo expansion and activation of patient-derived peripheral blood mononuclear cells (PBMCs) with a recombinant prostate-specific fusion protein (83). The registration trial involved CRPC patients and those receiving this treatment had in a median survival was 4.1 months longer than placebo-treated patients (83). Other additional immunotherapeutic approaches, including several vaccine trials including GVAX, and PROSTVAC, however did not demonstrate a survival benefit compared to placebo in phase 3 trials despite encouraging early results (84-86).

ICI treatment in PCa has to date demonstrated less than exciting results; a Phase III trial testing CTLA-4 blockade (Ipilimumab) did not observe any differences in overall survival compared to placebo in CRPC patients (87). Ipilimumab, analyzed in two Phase III studies, did not show any survival benefit in this tumor. The KEYNOTE-199 study analyzed the role of pembrolizumab for post-docetaxel mCRPC patients and concluded that pembrolizumab had antitumor activity and acceptable safety in these patients (88). Its activity was observed both in PD-L1 positive and PD-L1 negative cohorts, however, the response rate was low, with a complete and partial response of $<5 \%$ (88). To date, immune checkpoint inhibitors have yet to be FDA-approved for the management of metastatic PCa (86).

These and other data suggest that ICI alone may not be enough to facilitate a robust anti-tumor immune response in $\mathrm{PCa}$ patients, rather, activating tumor-specific TILs may provide more benefit. Future clinical trials investigating these agents should be encouraged on specific patient subsets including those with high PD-L1 expression, those with hypermutated or microsatelliteunstable tumors, and those enriched for germline and/or somatic DNA-repair gene mutations (e.g., intraductal/ductal histology, primary Gleason pattern 5, and perhaps AR-V7-positive tumors). Furthermore, neoadjuvant treatments which promote the development of an immunoreactive TIME could increase the sensitivity of CRPC patients to ICI and immunotherapy.

As the PCa TIME is usually non-inflamed and dominated by immunosuppressive cells, targeting or reprogramming these suppressive cell populations could skew the PCa TIME toward an inflamed phenotype and make PCa amenable for immunotherapy treatments. Accordingly, neoadjuvant administration of IFN1 agonists which activate cytosolic innate immune sensing pathways such as those mediated TLRs or STING, represents an area of unrealized potential in immunotherapy research for PCa. Preclinical findings have been promising; for example, the addition of intra-tumoral STING agonist injection to combination ICI treatment in a

\section{REFERENCES}

1. Wilt TJ, Brawer MK, Jones KM, Barry MJ, Aronson WJ, Fox S, et al. Radical prostatectomy versus observation for localized prostate cancer. N Engl J Med. (2012) 367:203-13. doi: 10.1056/NEJMoa1113162

2. Siegel RL, Miller KD, Jemal A. Cancer statistics, 2017. CA Cancer J Clin. (2017) 67:7-30. doi: 10.3322/caac.21387

3. Siegel RL, Miller KD, Jemal A. Cancer statistics, 2018. CA Cancer J Clin. (2018) 68:7-30. doi: 10.3322/caac. 21442 syngeneic mouse model of PCa increased overall survival by $35 \%$ compared to combination ICI alone (89). In this study, mice treated with both STING agonist and combination ICI had increased TIL: Treg and TIL: macrophage proportions, and decreased percentages of TAMs (89). Furthermore, it was demonstrated that this activation was not limited to STING agonists; poly I:C treatment in a syngeneic PCa mouse model has also shown to increase cellular differentiation and promote immunologically active lymphocyte infiltration (90). A more comprehensive understanding of the factors conferring sensitivity to IFN1 agonists is warranted as this approach moves forward. Discerning the immune pathways and mechanisms which significantly contribute to causing an inflamed and immunologically active TIME is required before these pathways can be therapeutically exploited. Finally, more trials, such as the recently initiated Quick efficacy seeking trial (Quest1) (86), are needed to determine precise immunotherapy combinations in PCa.

\section{CONCLUSIONS AND FUTURE PERSPECTIVES}

A detailed analysis of treatment naïve and treatment associated TIME is not currently available in PCa with reports to date mainly focusing on evaluation of limited phenotypes of activated or dysfunctional immune cell types. Sex-steroids, primarily androgens, play important roles in thymic involution or rejuvenation and thus therapeutic ablation of these could have significant impacts on the PCa TIME. The unique clinical and molecular features of each PCa case make it difficult to predict the status of the TIME, although some metrics such as TGF $\beta$ signaling and Treg infiltration may be useful. Importantly, use of genetic alterations such as PTEN loss and DDR status should be incorporated in trial design and accompany retrospective and prospective immune monitoring correlative studies.

\section{AUTHOR CONTRIBUTIONS}

MK and DS conceptualized the review. NV, SN, MK, and DS wrote and reviewed the review. NV and SN generated the figures.

\section{FUNDING}

This work was supported by grant support from Prostate Cancer Canada to MK and DS. The grant number D2016-1391.

4. Heidenreich A, Bastian PJ, Bellmunt J, Bolla M, Joniau S, van der Kwast $\mathrm{T}$, et al. EAU guidelines on prostate cancer. Part 1: screening, diagnosis, and local treatment with curative intent update 2013. Eur Urol. (2014) 65:124-37. doi: 10.1016/j.eururo.2013. 09.046

5. Freedland SJ, Humphreys EB, Mangold LA, Eisenberger M, Dorey FJ, Walsh PC, et al. Risk of prostate cancer-specific mortality following biochemical recurrence after radical prostatectomy. J Am Med Assoc. (2013) 294:433-9. doi: $10.1001 /$ jama.294.4.433 
6. Huggins C, Hodges CV. Studies on prostatic cancer: I. The effect of castration, of estrogen and of androgen injection on serum phosphatases in metastatic carcinoma of the prostate. CA Cancer J Clin. (1972) 22:232-40.

7. Chandrasekar T, Yang JC, Gao AC, Evans CP. Mechanisms of resistance in castration-resistant prostate cancer (CRPC). Trans Androl Urol. (2015) 4:365-80. doi: 10.3978/j.issn.2223-4683.2015.05.02

8. Gibney GT, Weiner LM, Atkins MB. Predictive biomarkers for checkpoint inhibitor-based immunotherapy. Lancet Oncol. (2016) 17:e542-51. doi: 10.1016/S1470-2045(16)30406-5

9. Spranger S, Gajewski TF. Tumor-intrinsic oncogene pathways mediating immune avoidance. Oncoimmunology. (2016) 5:e1086862. doi: 10.1080/2162402X.2015.1086862

10. Thorsson V, Gibbs DL, Brown SD, Wolf D, Bortone DS, Ou Yang TH, et al. The immune landscape of cancer. Immunity. (2018) 48:812-30.e14. doi: 10.1016/j.immuni.2018.03.023

11. Mouw KW, Goldberg MS, Konstantinopoulos PA, D'Andrea AD. DNA damage and repair biomarkers of immunotherapy response. Cancer Discov. (2017) 7:675-93. doi: 10.1158/2159-8290.CD-17-0226

12. Dallos MC, Drake CG. Blocking PD-1/PD-L1 in genitourinary malignancies. Cancer J. (2018) 24:20-30. doi: 10.1097/PPO.00000000000 00302

13. Fridman WH, Zitvogel L, Sautès-Fridman C, Kroemer G. The immune contexture in cancer prognosis and treatment. Nat Rev Clin Oncol. (2017) 14:717-34. doi: 10.1038/nrclinonc.2017.101

14. Galon J, Pagès F, Marincola FM, Angell HK, Thurin M, Lugli A, et al. Cancer classification using the immunoscore: a worldwide task force. J Trans Med. (2012) 10:205. doi: 10.1186/1479-5876-10-20

15. Danaher P, Warren S, Lu R, Samayoa J, Sullivan A, Pekker I, et al. Pan-cancer adaptive immune resistance as defined by the Tumor Inflammation Signature (TIS): results from The Cancer Genome Atlas (TCGA). J Immunother Cancer. (2018) 6:63. doi: 10.1186/s40425-018-0367-1

16. Shien K, Papadimitrakopoulou VA, Wistuba II. Predictive biomarkers of response to $\mathrm{PD}-1 / \mathrm{PD}-\mathrm{L} 1$ immune checkpoint inhibitors in non-small cell lung cancer. Lung Cancer. (2016) 99:79-87. doi: 10.1016/j.lungcan.2016.06.016

17. Balkwill FR, Capasso M, Hagemann T. The tumor microenvironment at a glance. J Cell Sci. (2012) 125:5591-6. doi: 10.1242/jcs.116392

18. Mellman I, Coukos G, Dranoff G. Cancer immunotherapy comes of age. Nature. (2011) 480:480-9. doi: 10.1038/nature10673

19. Topalian SL, Taube JM, Anders RA, Pardoll DM. Mechanism-driven biomarkers to guide immune checkpoint blockade in cancer therapy. Nat Rev Cancer. (2016) 16:275-87. doi: 10.1038/nrc.2016.36

20. Leclerc BG, Charlebois R, Chouinard G, Allard B, Pommey S, Saad F, et al. CD73 expression is an independent prognostic factor in prostate cancer. Clin Cancer Res. (2015) 22:158-67. doi: 10.1158/1078-0432.CCR-15-1181

21. Ness N, Andersen S, Valkov A, Nordby Y, Donnem T, Al-Saad S, et al. Infiltration of CD8+ lymphocytes is an independent prognostic factor of biochemical failure-free survival in prostate cancer. Prostate. (2014) 74:1452-61. doi: 10.1002/pros.22862

22. Petitprez F, Fossati N, Vano Y, Freschi M, Becht E, Lucianò R, et al. PD-L1 expression and CD8 $+\mathrm{T}$-cell infiltrate are associated with clinical progression in patients with node-positive prostate cancer. Eur Urol Focus. (2017) 8-12. doi: 10.1016/j.euf.2017.05.013

23. Zhao SG, Lehrer J, Chang SL, Das R, Erho N, Liu Y, et al. The immune landscape of prostate cancer and nomination of PD-L2 as a potential therapeutic target. J Natl Cancer Inst. (2018) 111:1-10. doi: 10.1093/jnci/djy141

24. Sharma P, Hu-Lieskovan S, Wargo JA, Ribas A. Primary, adaptive, and acquired resistance to cancer immunotherapy. Cell. (2017) 168:707-23. doi: 10.1016/j.cell.2017.01.017

25. Apetoh L, Smyth MJ, Drake CG, Abastado JP, Apte RN, Ayyoub M, et al. Consensus nomenclature for CD8 $+\mathrm{T}$ cell phenotypes in cancer. Oncoimmunology. (2015) 4:e998538. doi: 10.1080/2162402X.2014.998538

26. Kiniwa Y, Miyahara Y, Wang HY, Peng W, Peng G, Wheeler TM, et al. CD8+ Foxp3+ regulatory $\mathrm{T}$ cells mediate immunosuppression in prostate cancer. Clin Cancer Res. (2007) 13:6947-58. doi: 10.1158/1078-0432.CCR-07-0842

27. Kaur HB, Guedes LB, Lu J, Maldonado L, Reitz L, Barber JR, et al. Association of tumor-infiltrating T-cell density with molecular subtype, racial ancestry and clinical outcomes in prostate cancer. Mod Pathol. (2018) 31:1539-52. doi: 10.1038/s41379-018-0083-x

28. Nardone V, Botta C, Caraglia M, Martino EC, Ambrosio MR, Carfagno $\mathrm{T}$, et al. Tumor infiltrating $\mathrm{T}$ lymphocytes expressing FoxP3, CCR7 or PD-1 predict the outcome of prostate cancer patients subjected to salvage radiotherapy after biochemical relapse. Cancer Biol Ther. (2016) 17:1213-20. doi: 10.1080/15384047.2016.1235666

29. Watanabe M, Kanao K, Suzuki S, Muramatsu H, Morinaga S, Kajikawa K, et al. Abstract 3961: increased CCR4-positive regulatory $\mathrm{T}$ cells in biopsy specimen of poor prognosis prostate cancer. Cancer Res. (2017) 77:3961. doi: 10.1158/1538-7445.AM2017-3961

30. Won KY, Kim GY, Kim HK, Choi SI, Kim SH, Bae GE, et al. Tumoral FOXP3 expression is associated with favorable clinicopathological variables and good prognosis in gastric adenocarcinoma: the tumor suppressor function of tumoral FOXP3 is related with the P21 expression in gastric adenocarcinoma. Hum Pathol. (2017) 68:112-8. doi: 10.1016/j.humpath.2017.08.023

31. Lundholm M, Hägglöf C, Wikberg ML, Stattin P, Egevad L, Bergh A, et al. Secreted factors from colorectal and prostate cancer cells skew the immune response in opposite directions. Sci Rep. (2015) 5:15651. doi: $10.1038 /$ srep 15651

32. Mariathasan S, Turley SJ, Nickles D, Castiglioni A, Yuen K, Wang Y, et al. TGF $\beta$ attenuates tumour response to PD-L1 blockade by contributing to exclusion of T cells. Nature. (2018) 554:544-8. doi: 10.1038/nature25501

33. Bryant G, Wang L, Mulholland DJ. Overcoming oncogenic mediated tumor immunity in prostate cancer. Int J Mol Sci. (2017) 18:E1542. doi: $10.3390 /$ ijms 18071542

34. Lawrence MS, Stojanov P, Polak P, Kryukov GV, Cibulskis K, Sivachenko A, et al. Mutational heterogeneity in cancer and the search for new cancerassociated genes. Nature. (2013) 499:214-8. doi: 10.1038/nature12213

35. Berger MF, Lawrence MS, Demichelis F, Drier Y, Cibulskis K, Sivachenko AY, et al. The genomic complexity of primary human prostate cancer. Nature. (2011) 470:214-20. doi: 10.1038/nature09744

36. Schreiber RD, Old LJ, Smyth MJ. Cancer immunoediting: integrating immunity's roles in cancer suppression and promotion. Science. (2011) 331:1565-70. doi: 10.1126/science. 1203486

37. Tran E, Robbins PF, Rosenberg SA. 'Final common pathway' of human cancer immunotherapy: targeting random somatic mutations. Nat Immunol. (2017) 18:255-62. doi: $10.1038 /$ ni.3682

38. O'Connor MJ. Targeting the DNA damage response in cancer. Mol Cell. (2015) 60:547-60. doi: 10.1016/j.molcel.2015.10.040

39. Buckley NE, Hosey AM, Gorski JJ, Purcell JW, Mulligan JM, Harkin DP, et al. BRCA1 regulates IFN- signaling through a mechanism involving the type I IFNs. Mol Cancer Res. (2007) 5:261-70. doi: 10.1158/1541-7786.MCR-06-0250

40. Abeshouse A, Ahn J, Akbani R, Ally A, Amin S, Andry CD, et al. The molecular taxonomy of primary prostate cancer. Cell. (2015) 163:1011-25. doi: 10.1016/j.cell.2015.10.025

41. Robinson D, Van Allen EM, Wu YM, Schultz N, Lonigro RJ, Mosquera JM, et al. Integrative clinical genomics of advanced prostate cancer. Cell. (2015) 161:1215-28. doi: 10.1016/j.cell.2015.05.001

42. Mateo J, Carreira S, Sandhu S, Miranda S, Mossop H, Perez-Lopez R, et al. DNA-repair defects and olaparib in metastatic prostate cancer. $\mathrm{N} \mathrm{Engl} \mathrm{J} \mathrm{Med.}$ (2015) 373:1697-708. doi: 10.1056/NEJMoa1506859

43. Pritchard CC, Mateo J, Walsh MF, De Sarkar N, Abida W, Beltran H, et al. Inherited DNA-repair gene mutations in men with metastatic prostate cancer. N Engl J Med. (2016) 375:443-53. doi: 10.1056/NEJMoa1603144

44. Goodwin JF, Schiewer MJ, Dean JL, Schrecengost RS, de Leeuw R, Han S, et al. A hormone-DNA repair circuit governs the response to genotoxic insult. Cancer Discov. (2013) 3:1254-71. doi: 10.1158/2159-8290.CD-13-0108

45. Polkinghorn WR, Parker JS, Lee MX, Kass EM, Spratt DE, Iaquinta PJ, et al. Androgen receptor signaling regulates DNA repair in prostate cancers. Cancer Discov. (2013) 3:1245-53. doi: 10.1158/2159-8290.CD-13-0172

46. Teo MY, Seier K, Ostrovnaya I, Regazzi AM, Kania BE, Moran MM, et al. Alterations in DNA damage response and repair genes as potential marker of clinical benefit from PD-1/PD-L1 blockade in advanced urothelial cancers. $J$ Clin Oncol. (2018) 36:1685-94. doi: 10.1200/JCO.2017.75.7740

47. Garcia-Lora A, Algarra I, Garrido F. MHC class I antigens, immune surveillance, and tumor immune escape. J Cell Physiol. (2003) 195:346-55. doi: $10.1002 /$ jcp. 10290 
48. Heninger E, Krueger TE, Thiede SM, Sperger JM, Byers BL, Kircher MR, et al. Inducible expression of cancer-testis antigens in human prostate cancer. Oncotarget. (2016) 7:84359-74. doi: 10.18632/oncotarget.12711

49. Sanda MG, Restifo NP, Walsh JC, Kawakami Y, Nelson WG, Pardoll DM, et al. Molecular characterization of defective antigen processing in human prostate cancer. J Natl Cancer Inst. (1995) 87:280-5. doi: 10.1093/jnci/87.4.280

50. Bander NH, Yao D, Liu H, Chen YT, Steiner M, Zuccaro W, et al. MHC class I and II expression in prostate carcinoma and modulation by interferon-alpha and -gamma. Prostate. (1997) 33:233-9.

51. Martini M, Testi MG, Pasetto M, Picchio MC, Innamorati G, Mazzocco M, et al. IFN- $\gamma$-mediated upmodulation of MHC class I expression activates tumor-specific immune response in a mouse model of prostate cancer. Vaccine. (2010) 28:3548-57. doi: 10.1016/j.vaccine.2010.03.007

52. Reits EA, Hodge JW, Herberts CA, Groothuis TA, Chakraborty M, Wansley EK, et al. Radiation modulates the peptide repertoire, enhances MHC class I expression, and induces successful antitumor immunotherapy. J Exp Med. (2006) 203:1259-71. doi: 10.1084/jem.20052494

53. Stambolic V, Suzuki A, de la Pompa JL, Brothers GM, Mirtsos C, Sasaki T, et al. Negative regulation of $\mathrm{PKB} /$ Akt-dependent cell survival by the tumor suppressor PTEN. Cell. (1998) 95:29-39. doi: 10.1016/S0092-8674(00)81780-8

54. Chen L, Guo D. The functions of tumor suppressor PTEN in innate and adaptive immunity. Cell Mol Immunol. (2017) 14:581-9. doi: $10.1038 / \mathrm{cmi} .2017 .30$

55. Peng W, Chen JQ, Liu C, Malu S, Creasy C, Tetzlaff MT, et al. Loss of PTEN promotes resistance to T cell-mediated immunotherapy. Cancer Discov. (2016) 6:202-16. doi: 10.1158/2159-8290.CD-15-0283

56. Bezzi M, Seitzer N, Ishikawa T, Reschke M, Chen M, Wang G, et al. Diverse genetic-driven immune landscapes dictate tumor progression through distinct mechanisms. Nat Med. (2018) 24:165-75. doi: 10.1038/nm.4463

57. Bassi C, Ho J, Srikumar T, Dowling RJ, Gorrini C, Miller SJ, et al. Nuclear PTEN controls DNA repair and sensitivity to genotoxic stress. Science. (2013) 341:395-9. doi: 10.1126/science. 1236188

58. Shen SM, Ji Y, Zhang C, Dong SS, Yang S, Xiong Z, et al. Nuclear PTEN safeguards pre-mRNA splicing to link Golgi apparatus for its tumor suppressive role. Nat Commun. (2018) 9:2392. doi: 10.1038/s41467-018-04760-1

59. Lee YR, Chen M, Pandolfi PP. The functions and regulation of the PTEN tumour suppressor: new modes and prospects. Nat Rev Mol Cell Biol. (2018) 19:547-62. doi: 10.1038/s41580-018-0015-0

60. Coccia EM, Severa M, Giacomini E1, Monneron D, Remoli ME, Julkunen I, et al. Viral infection and toll-like receptor agonists induce a differential expression of type I and lamda interferons in human plasmacytoid and monocyte-derived dendritic cells. Eur J Immunol. (2004) 34:796-805. doi: 10.1002/eji.200324610

61. Theofilopoulos AN, Baccala R, Beutler B, Kono DH. Type I Interferons $(\alpha / \beta)$ in immunity and autoimmunity. Annu Rev Immunol. (2005) 23:307-36. doi: 10.1146/annurev.immunol.23.021704.115843

62. Ivashkiv LB, Donlin LT. Regulation of type I interferon responses. Nat Rev Immunol. (2013) 14:36-49. doi: 10.1038/nri3581

63. Pencik J, Schlederer M, Gruber W, Unger C, Walker SM, Chalaris A, et al. STAT3 regulated ARF expression suppresses prostate cancer metastasis. Nat Commun. (2015) 6:7736. doi: 10.1038/ncomms8736

64. Lee SO, Lou W, Qureshi KM, Mehraein-Ghomi F, Trump DL, Gao AC. RNA interference targeting Stat3 inhibits growth and induces apoptosis of human prostate cancer cells. Prostate. (2004) 60:303-9. doi: 10.1002/pros.20072

65. Canesin G, Evans-Axelsson S, Hellsten R, Sterner O, Krzyzanowska A, Andersson T, et al. The STAT3 inhibitor galiellalactone effectively reduces tumor growth and metastatic spread in an orthotopic xenograft mouse model of prostate cancer. Eur Urol. (2016) 69:400-4. doi: 10.1016/j.eururo.2015.06.016

66. Cheon H, Stark GR. Unphosphorylated STAT1 prolongs the expression of interferon-induced immune regulatory genes. Proc Natl Acad Sci USA. (2009) 106:9373-8. doi: 10.1073/pnas.0903487106

67. Yang J, Chatterjee-Kishore M, Staugaitis SM, Nguyen H, Schlessinger K, Levy DE, et al. Novel roles of unphosphorylated STAT3 in oncogenesis and transcriptional regulation. Cancer Res. (2005) 65:939-47.

68. Minn AJ. Interferons and the immunogenic effects of cancer therapy. Trends Immunol. (2015) 36:725-37. doi: 10.1016/j.it.2015.09.007
69. Cheon H, Borden EC, Stark GR. Interferons and their stimulated genes in the tumor microenvironment. Semin Oncol. (2014) 41:156-73. doi: 10.1053/j.seminoncol.2014.02.002

70. Saad F, Fizazi K. Androgen deprivation therapy and secondary hormone therapy in the management of hormone-sensitive and castration-resistant prostate cancer. Urology. (2015) 86:852-61. doi: 10.1016/j.urology.2015.07.034

71. $\mathrm{Pu} \mathrm{Y,} \mathrm{Xu} \mathrm{M,} \mathrm{Liang} \mathrm{Y,} \mathrm{Yang} \mathrm{K}$, Guo $\mathrm{Y}$, Yang $\mathrm{X}$, et al. Androgen receptor antagonists compromise $\mathrm{T}$ cell response against prostate cancer leading to early tumor relapse. Sci Trans Med. (2016) 8:333ra47. doi: 10.1126/scitranslmed.aad5659

72. Kroemer G, Galluzzi L, Kepp O, Zitvogel L. Immunogenic cell death in cancer therapy. Annu Rev Immunol. (2013) 31:51-72. doi: 10.1146/annurev-immunol-032712-100008

73. Kalina JL, Neilson DS, Comber AP, Rauw JM, Alexander AS, Vergidis J, et al. Immune modulation by androgen deprivation and radiation therapy: implications for prostate cancer immunotherapy. Cancers (Basel). (2017) 9:E13. doi: 10.3390/cancers9020013

74. Tang S, Moore ML, Grayson JM, Dubey P. Increased CD8+ Tcell function following castration and immunization is countered by parallel expansion of regulatory T cells. Cancer Res. (2012) 72:1975-85. doi: 10.1158/0008-5472.CAN-11-2499

75. Escamilla J, Schokrpur S, Liu C, Priceman SJ, Moughon D, Jiang Z, et al. CSF1 receptor targeting in prostate cancer reverses macrophage-mediated resistance to androgen blockade therapy. Cancer Res. (2015) 75:950-62. doi: 10.1158/0008-5472.CAN-14-0992

76. Nonomura N, Takayama H, Nakayama M, Nakai Y, Kawashima A, Mukai $\mathrm{M}$, et al. Infiltration of tumour-associated macrophages in prostate biopsy specimens is predictive of disease progression after hormonal therapy for prostate cancer. BJU Int. (2011) 107:1918-22. doi: 10.1111/j.1464-410X.2010.09804.x

77. Fucikova J, Kralikova P, Fialova A, Brtnicky T, Rob L, Bartunkova J, et al. Human tumor cells killed by anthracyclines induce a tumor-specific immune response. Cancer Res. (2011) 71:4821-33. doi: 10.1158/0008-5472.CAN-11-0950

78. DeNardo DG, Brennan DJ, Rexhepaj E, Ruffell B, Shiao SL, Madden SF, et al. Leukocyte complexity predicts breast cancer survival and functionally regulates response to chemotherapy. Cancer Discov. (2011) 1:5467. doi: 10.1158/2159-8274.CD-10-0028

79. Hodge JW, Garnett CT, Farsaci B, Palena C, Tsang KY, Ferrone S, et al. (2013).Chemotherapy-induced immunogenic modulation of tumor cells enhances killing by cytotoxic $\mathrm{T}$ lymphocytes and is distinct from immunogenic cell death. Int J Cancer. 133:624-36. doi: 10.1002/ijc.28070

80. Kodumudi KN, Woan K, Gilvary DL, Sahakian E, Wei S, Djeu JY. A novel chemoimmunomodulating property of docetaxel: suppression of myeloidderived suppressor cells in tumor bearers. Clin Cancer Res. (2010) 16:4583-94. doi: 10.1158/1078-0432.CCR-10-0733

81. Arlen PM, Gulley JL, Parker C, Skarupa L, Pazdur M, Panicali D, et al. A randomized phase II study of concurrent docetaxel plus vaccine versus vaccine alone in metastatic androgen-independent prostate cancer. Clin Cancer Res. (2006) 12:1260-9. doi: 10.1158/1078-0432.CCR-05-2059

82. Cho Y, Chang JS, Rha KH, Hong SJ, Choi YD, Ham WS, et al. Does radiotherapy for the primary tumor benefit prostate cancer patients with distant metastasis at initial diagnosis? PLoS ONE. (2016) 11:e0147191. doi: 10.1371/journal.pone.0147191

83. Kantoff PW, Higano CS, Shore ND, Berger ER, Small EJ, Penson D F, et al. Sipuleucel-T immunotherapy for castration-resistant prostate cancer. $N$ Engl J Med. (2010) 363:411-22. doi: 10.1056/NEJMoa1001294

84. Singh P, Pal SK, Alex A, Agarwal N. Development of PROSTVAC immunotherapy in prostate cancer. Future Oncol. (2015) 11:2137-48. doi: $10.2217 /$ fon. 15.120

85. Redman JM, Gulley JL, Madan RA. Combining immunotherapies for the treatment of prostate cancer. Urol Oncol Semin Orig Investig. (2017) 35:694700. doi: 10.1016/j.urolonc.2017.09.024

86. Redman JM, Steinberg SM, Gulley JL. Quick efficacy seeking trial (QuEST1): a novel combination immunotherapy study designed for rapid clinical signal assessment metastatic castration-resistant prostate cancer. J Immunother Cancer. (2018) 6:91. doi: 10.1186/s40425-018-0409-8 
87. Kwon ED, Drake CG, Scher HI, Fizazi K, Bossi A, van den Eertwegh AJ, et al. Ipilimumab versus placebo after radiotherapy in patients with metastatic castration-resistant prostate cancer that had progressed after docetaxel chemotherapy (CA184-043): a multicentre, randomised, double-blind, phase 3 trial. Lancet Oncol. (2014) 15:700-12. doi: 10.1016/S1470-2045(14)70189-5

88. De Bono JS, Goh JC, Ojamaa K, Piulats Rodriguez JM, Drake CG, Hoimes CJ, et al. KEYNOTE-199: Pembrolizumab (pembro) for docetaxel-refractory metastatic castration-resistant prostate cancer (mCRPC). J Clin Oncol. (2018) 36:5007. doi: 10.1200/JCO.2018.36.15_suppl.5007

89. Ager CR, Reilley MJ, Nicholas C, Bartkowiak T, Jaiswal AR, Curran MA. Intratumoral STING activation with T-cell checkpoint modulation generates systemic antitumor immunity. Cancer Immunol Res. (2017) 5:676-84. doi: 10.1158/2326-6066.CIR-17-0049

90. Chin AI, Miyahira AK, Covarrubias A, Teague J, Guo B, Dempsey PW, et al. Toll-like receptor 3-mediated suppression of TRAMP prostate cancer shows the critical role of type I interferons in tumor immune surveillance. Cancer Res. (2010) 70:2595-603. doi: 10.1158/0008-5472. CAN-09-1162

Conflict of Interest Statement: The authors declare that the research was conducted in the absence of any commercial or financial relationships that could be construed as a potential conflict of interest.

Copyright $(02019$ Vitkin, Nersesian, Siemens and Koti. This is an open-access article distributed under the terms of the Creative Commons Attribution License (CC BY). The use, distribution or reproduction in other forums is permitted, provided the original author(s) and the copyright owner(s) are credited and that the original publication in this journal is cited, in accordance with accepted academic practice. No use, distribution or reproduction is permitted which does not comply with these terms. 